\title{
Casimir bag energy in the stochastic approximation to the pure QCD vacuum
}

\author{
C. D. Fosco ${ }^{a}$ and L. E. Oxman ${ }^{b}$ \\ ${ }^{a}$ Centro Atómico Bariloche and Instituto Balseiro \\ Comisión Nacional de Energía Atómica \\ R8402AGP Bariloche, Argentina. \\ ${ }^{b}$ Instituto de Física, Universidade Federal Fluminense \\ Av. Litorânea $S / N$, Boa Viagem, \\ Niterói, RJ 24210-340, Brazil.
}

PACS: 12.39.Ba, 12.38.Lg

Keywords: Casimir energy, bag model, nonperturbative QCD.

\begin{abstract}
We study the Casimir contribution to the bag energy coming from gluon field fluctuations, within the context of the stochastic vacuum model (SVM) of pure QCD. After formulating the problem in terms of the generating functional of field strength cumulants, we argue that the resulting predictions about the Casimir energy are compatible with the phenomenologically required bag energy term.
\end{abstract}

\section{Introduction}

In order to adjust hadron spectroscopy, the MIT bag model requires a bag energy term of the form $-Z / R$ with a positive $Z$ of order one [1]. One of the main contributions to this energy is naturally ascribed to the Casimir energy of the fields involved. However, due to the daunting difficulty posed by Casimir energy calculations in nonabelian gauge field theories, this problem has only been approached under rather strong simplifying assumptions. Indeed, vacuum effects coming from the pure Yang-Mills sector have been computed by considering a simplified description, which treats gluon modes 
as a collection of free, photon-like gauge fields. This assumption (usually justified by invoking asymptotic freedom) leads, however, to an energy with the wrong sign: $\sim+0.7 / R$. Thus the effect becomes repulsive, instead of the attractive one needed to comply with phenomenology [2]-[6]. For a discussion of the bag model, as well as of the current status of Casimir calculations in the bag, see, for example, refs. [7] and [8].

Neither the inclusion of the (rather small) fermionic Casimir energy contribution $\sim-0.02 / R$ due to three light quark modes [9], nor the (relevant) effect of the quarks' center of mass quantum fluctuations, which is attractive, $\sim-Z_{c m} / R, Z_{c m} \sim 0.6-0.8$, are sufficient to reach the required value of $Z$ (see the discussion in [7] and [10]).

It has been pointed out that a resolution to this problem could be associated with the intrinsically nonabelian nature of the Yang-Mills theories, and their ensuing nonperturbative effects. This is certainly plausible, although it is not at all evident how to include those effects in a tractable way.

In this article, we present an approach that enables one to take partly into account the nonperturbative dynamics of QCD into the calculation of the Casimir energy, and argue that its predictions are phenomenologically sound.

We first show how the Casimir energy in pure QCD can be written in terms of a subclass of gauge invariant correlators, namely, the shifted fieldstrength correlators. Lattice simulations show [11 that these correlators exhibit Gaussian dominance, so that they can be described in terms of the so called stochastic vacuum model (SVM) of pure QCD [12] (for a review see refs. [13] and [14]). This model can be parametrized in terms of just two structure functions, obtained by fitting the lattice data, and it consistently describes short distance perturbative aspects, such as asymptotic freedom, as well as long distance nonperturbative ones, such as confinement.

In order to relate Casimir energy calculations to correlators of the stochastic model, we will use a framework where the boundary conditions are imposed by means of auxiliary fields living on the bag boundary. In this way, we are able to show that the lattice-adjusted SVM parameters imply that the simple model that computes Casimir energies treating gluon modes as massless photon-like gauge fields is not reliable; rather, a scenario with a suppressed Casimir energy due to gapped nonperturbative effective modes is favoured. Thus, a physical picture arises where the suppression of the gluon Casimir energy leaves room for the effect of center of mass quantum fluctuations to dominate the $R^{-1}$ contribution to the bag energy.

The structure of this paper is as follows: in section 2, as a preparation for the nonabelian case, we use the approach of [15] to construct the path integral for the Casimir energy for a confined Abelian gauge field with magnetic 
boundary conditions, in terms of the field-strength correlation function. In section 3, the same approach is followed for a nonabelian gauge field within the context of the SVM. In section 4 , we show that the same vacuum energy that one obtains from the SVM may be alternatively understood as due to a pair of fields, one of them vectorial and the other pseudovectorial, both endowed with non-standard kinetic terms. This parametrization is then applied to infer the main properties of the SVM Casimir energy.

In section 6, we present our conclusions.

\section{Confined Abelian field}

Let us first consider an Abelian gauge field $A_{\mu}$ in $3+1$ dimensions, confined to a spatial region $\mathcal{V}$ whose boundary is a regular (static) surface $\Sigma$. On that surface, we shall consider boundary conditions corresponding to a perfect conductor of magnetic charges.

We shall assume that $\Sigma$ can be described in parametric form,

$$
\begin{aligned}
\mathbf{r}: U & \longrightarrow \mathbb{R}^{3} \\
\left(\zeta^{1}, \zeta^{2}\right) & \longrightarrow \mathbf{r}\left(\zeta^{1}, \zeta^{2}\right)
\end{aligned}
$$

where $U$ is a finite region of $\mathbb{R}^{2}$, the domain of the parameters $\zeta^{1}, \zeta^{2}$. In order the impose the boundary conditions, it is convenient to introduce first some notation and conventions. Since the boundary conditions are static, they shall hold on the world-volume $\Gamma=\mathbb{R} \times \Sigma$ swept by $\Sigma$ in the course of its (trivial) time evolution. This world-volume can be parametrized by a function $r_{\mu}(\mu=0,1,2,3)$, depending on $\zeta^{1}, \zeta^{2}$ as well as on an extra parameter $\zeta^{0}$ :

$$
\begin{aligned}
r_{\mu}: \mathbb{R} \times U & \longrightarrow \mathbb{R}^{4} \\
\left(\zeta^{0}, \zeta^{1}, \zeta^{2}\right) & \longrightarrow r_{\mu}(\zeta),
\end{aligned}
$$

where $r_{0}(\zeta)=\zeta_{0}$ and $r_{i}(\zeta)=r_{i}\left(\zeta_{1}, \zeta_{2}\right)$.

Regarding the 'target' space, we shall work in Euclidean spacetime, with coordinates $x_{\mu}, \mu=0,1,2,3$, and a metric which is the identity matrix (no special meaning will be given to the position -up or down- of space and spacetime indices). Letters from the beginning of the Greek alphabet run from 0 to 2 , those from the middle do so from 0 to 3 , while Roman ones can take values from 1 to 3 . Besides, $a, b, c, \ldots$ will be reserved for surface coordinates, and therefore may assume the values 1 or 2 . 
At any point of $\Gamma$, we have the three tangent vectors $t_{\alpha}^{\mu}=\frac{\partial r^{\mu}}{\partial \zeta^{\alpha}}$, which are not necessarily orthogonal but certainly linearly independent vectors 1 . Of course, $t_{1}$ and $t_{2}$ are tangent to $\Sigma$, at all times.

Besides, we note that each component $t^{\mu}$ of $t$ transforms as a covariant vector under reparametrizations: $t_{\alpha}^{\prime}\left(\zeta^{\prime}\right)=\frac{\partial \zeta^{\beta}}{\partial \zeta^{\prime \alpha}} t_{\beta}(\zeta)$.

Finally, we shall also make use of $\mathbf{n}$, the (outer) normal to $\Sigma$. At any point $\mathbf{r}(\zeta)$ it can be easily obtained as the vector product of the two tangent vectors $t_{1}$ and $t_{2}: \mathbf{n}=\mathbf{t}_{1} \times \mathbf{t}_{2}$. This vector may be easily normalized by taking into account the fact that:

$$
\mathbf{n}^{2}(\zeta)=\left(\mathbf{t}_{1}(\zeta) \times \mathbf{t}_{2}(\zeta)\right)^{2}=g_{\Sigma}(\zeta)
$$

where $g_{\Sigma} \equiv \operatorname{det}\left(g_{a b}\right)$, and $g_{a b}$ is the induced metric on $\Sigma$ :

$$
g_{a b}(\zeta)=t_{a}^{\mu}(\zeta) t_{b}^{\mu}(\zeta)
$$

thus, the unit normal $\hat{\mathbf{n}}$ is simply: $\hat{\mathbf{n}} \equiv \frac{\mathbf{n}}{|\mathbf{n}|}=\frac{1}{\sqrt{g_{\Sigma}}} \mathbf{n}$. As a matter of fact, since the time evolution of the boundary is trivial, $g_{\Gamma}$, the determinant of the induced metric on $\Gamma$, coincides with $g_{\Sigma}$.

Regarding the transformation properties of $\hat{\mathbf{n}}$, one easily sees that it behaves as a pseudo-scalar under surface reparametrizations.

Now we introduce the magnetic boundary conditions: they amount to surrounding the region $\mathcal{V}$ with a perfect magnetic conductor, so that the tangential component of $\mathbf{B}$ at every point of $\Sigma$, and for every time vanishes:

$$
\hat{\mathbf{n}}(\zeta) \times \mathbf{B}\left(r_{\mu}(\zeta)\right)=\mathbf{0}
$$

or, in terms of $F_{\mu \nu}$,

$$
F_{i j}\left(r_{\mu}(\zeta)\right) \hat{n}^{j}(\zeta)=0, \quad i=1,2,3 .
$$

Note that they do not amount to three independent conditions, since they satisfy the condition: $\hat{n}_{i} F_{i j} \hat{n}_{j}=0$. Thus, (6) may be written equivalently as:

$$
\eta_{a} \equiv t_{a}^{i} F_{i j} \hat{n}^{j}=0, \quad a=1,2,
$$

where only the two independent constraints, $\eta_{1}$ and $\eta_{2}$ appear (note that $t_{0}^{i}=0$, thus there is no other constraint).

Let us now consider the Euclidean path integral that result from imposing the previously introduced constraints into the vacuum transition amplitude

\footnotetext{
${ }^{1}$ At least under the assumption that $\Sigma$ is a regular surface. They may be normalized, but that step shall not be necessary.
} 
for the Maxwell field. To that end, we shall define $\mathcal{Z}_{\text {mag }}$, the partition function corresponding to a magnetic charge conductor, since it is more similar to the case we will need to deal with in the nonabelian case:

$$
\begin{aligned}
\mathcal{Z}_{\text {mag }} & \equiv \int[\mathcal{D} A]_{\text {mag }} e^{-S_{g}[A]} \\
& =\mathcal{N} \int \mathcal{D} A \prod_{a=1}^{2} \delta\left[\eta_{a}\right] e^{-S_{g}[A]}
\end{aligned}
$$

where $S_{g}$ is the gauge field action (including gauge fixing), and $[\mathcal{D} A]_{\text {mag }}$ is the integration measure for the gauge field, assuming it satisfies the corresponding boundary conditions on $\Sigma$. Following [15], those conditions have been imposed, on the second line, by means of two $\delta$ functionals. The integration measure for $A_{\mu}$ becomes then unconstrained.

It is convenient to exponentiate the $\delta$ functionals, by means of two Lagrange multipliers, denoted by $\phi^{a}(\zeta)$. It has been shown that the exponentiated $\delta$-functionals have to be invariant under reparametrizations of the surface [15]. Thus, it is immediate to see that the proper expression is:

$$
\prod_{a=1}^{2} \delta\left[\eta_{a}\right]=\int \mathcal{D} \phi e^{-S_{\delta}[\phi, A]}
$$

where

$$
S_{\delta}[\phi, A]=-i \int d^{3} \zeta \sqrt{g_{\Gamma}(\zeta)} \phi^{a}(\zeta) \eta_{a}(\zeta)
$$

Reparametrization invariance of $S_{\delta}$ is easily verified; indeed, $d^{3} \zeta \sqrt{g_{\Gamma}(\zeta)}$ is invariant by itself, while $\eta_{a}$ is a covariant vector, whose change is compensated by the variation of the contravariant vector $\phi^{a}$. Note that the $\sqrt{g_{\Gamma}(\zeta)}=\sqrt{g_{\Sigma}(\zeta)}$ factor may be cancelled with a like one in the unit normal; thus:

$$
\begin{aligned}
S_{\delta}[\phi, A] & =-i \int d^{3} \zeta \phi^{a}(\zeta) t_{a}^{i}(\zeta) F_{i j}\left(r_{\mu}(\zeta)\right) n^{j}(\zeta) \\
& =-i \int d^{3} \zeta \phi^{a}(\zeta) \partial_{a} r^{i}(\zeta) F_{i j}\left(r_{\mu}(\zeta)\right) \epsilon^{j k l} \partial_{1} r^{k}(\zeta) \partial_{2} r^{l}(\zeta) .
\end{aligned}
$$

This term may be regarded as an interaction between the gauge field and a 'current' $J^{\Sigma}$ due to the boundary conditions:

$$
S_{\delta}=-\frac{i}{2} \int d^{4} x J_{k l}^{\Sigma}(x) F_{k l}(x)
$$


with

$$
J_{k l}^{\Sigma}(x)=\int d^{3} \zeta \phi^{a}(\zeta) \delta^{(4)}\left(x_{\mu}-r_{\mu}(\zeta)\right)\left[\left(\partial_{a} r_{k}\right) n_{l}-\left(\partial_{a} r_{l}\right) n_{k}\right](\zeta) .
$$

Thus, in terms of $\mathcal{Z}\left(J_{\mu \nu}\right)$, the generating functional of field strength correlators:

$$
\mathcal{Z}\left(J_{\mu \nu}\right) \equiv e^{-\mathcal{W}\left(J_{\mu \nu}\right)}=\frac{\int \mathcal{D} A e^{-S_{g}(A)+\frac{i}{2} \int d^{4} x J_{\mu \nu} F_{\mu \nu}}}{\int \mathcal{D} A e^{-S_{g}(A)}},
$$

$\mathcal{Z}_{\text {mag }}$ may be written as:

$$
\mathcal{Z}_{\text {mag }}=\left.\int \mathcal{D} \phi \exp \left[-\mathcal{W}\left(J_{\mu \nu}\right)\right]\right|_{J=J^{\Sigma}}
$$

where $J_{\mu \nu}^{\Sigma}$ agrees with (13) when both indices are spatial, and is assumed to vanish when one of them equals zero. Of course, the integral is Gaussian, and one is able to write the result:

$$
\mathcal{W}\left(J_{\mu \nu}\right)=\frac{1}{8} \int d^{4} x \int d^{4} y J_{\mu \nu}(x)\left\langle F_{\mu \nu}(x) F_{\rho \sigma}(y)\right\rangle J_{\rho \sigma}(y),
$$

where:

$$
\left\langle F_{\mu \nu}(x) F_{\rho \sigma}(y)\right\rangle \equiv \Delta_{\mu \nu, \rho \sigma}(x-y)
$$

with

$$
\Delta_{\mu \nu, \rho \sigma}(x)=\int \frac{d^{4} k}{(2 \pi)^{4}} e^{i k \cdot x} \tilde{\Delta}_{\mu \nu, \rho \sigma}(k)
$$

and

$$
\tilde{\Delta}_{\mu \nu, \rho \sigma}(k)=-\frac{1}{k^{2}}\left(\delta_{\nu \sigma} k_{\mu} k_{\rho}+\delta_{\mu \rho} k_{\nu} k_{\sigma}-\delta_{\mu \sigma} k_{\nu} k_{\rho}-\delta_{\nu \rho} k_{\mu} k_{\sigma}\right) .
$$

The coordinate-space version of (19) may be expressed as follows:

$$
\Delta_{\mu \nu, \rho \sigma}(z)=\frac{1}{2}\left[\partial_{\nu}\left(z_{\sigma} \delta_{\mu \rho}-z_{\rho} \delta_{\mu \sigma}\right)+\partial_{\mu}\left(z_{\rho} \delta_{\nu \sigma}-z_{\sigma} \delta_{\nu \rho}\right)\right] D_{0}\left(z^{2}\right)
$$

where

$$
z \equiv x-y, \quad D_{0}\left(z^{2}\right) \equiv \frac{1}{\pi^{2}} \frac{d}{d z^{2}} \frac{1}{z^{2}}=-\frac{1}{\pi^{2} z^{4}} .
$$

The vacuum energy $E_{0}$ in the presence of the boundary $\Sigma$ is then given by:

$$
E_{0}=-\lim _{T \rightarrow \infty} \frac{1}{T} \ln \mathcal{Z}_{m a g},
$$

where $T$ denotes the extension of the Euclidean time coordinate: $\left|x_{0}\right| \leq T / 2$. 


\section{Confined nonabelian gauge field}

For a region surrounded by a perfect conductor of chromomagnetic charges, we have at the boundary the condition:

$$
\mathbf{B} \times \hat{\mathbf{n}}=\mathbf{0}
$$

where $\mathbf{B} \equiv \mathbf{B}^{u} T_{u}$ and $T_{u}$ are the generators of the Lie group. The index $u$ runs from 1 to $N$, the dimension of the adjoint representation (i.e., $N=8$, for $S U(3))$. In terms of the nonabelian field strength,

$$
F_{i j}\left(r_{\mu}(\zeta)\right) \hat{n}_{j}(\zeta)=0
$$

As the constraints (24) are homogeneous, they can also be written in the equivalent form

$$
F_{i j}(y, x ; \mathcal{C}) \hat{n}_{j}(\zeta)=0, \quad \forall x \in \Sigma,
$$

obtained by covariantly shifting the field strength $F_{i j}$ from an arbitrary (fixed) reference point $y . F_{\mu \nu}(y, x ; \mathcal{C})$ is the shifted field strength, defined by,

$$
F_{\mu \nu}(y, x ; \mathcal{C})=V(y, x ; \mathcal{C}) F_{\mu \nu}(x) V^{-1}(y, x ; \mathcal{C}),
$$

where $V$ is the parallel transporter, from $x$ to $y$, along a path $\mathcal{C}$,

$$
V(y, x ; \mathcal{C})=\mathcal{P} \exp \left(-i g \int_{\mathcal{C}} d z^{\mu} A_{\mu}(z)\right),
$$

and $\mathcal{P}$ is the path ordering operator.

The Casimir energy obtained by imposing either the constraints (24) or the equivalent ones (25) should be the same, since they differ by a gauge transformation 2 .

Thus we will impose the constraints,

$$
\eta_{a}^{u} \equiv t_{a}^{i} \hat{n}^{j} F_{i j}^{u}(x, y ; \mathcal{C})=0 .
$$

Introducing, as in the previous section, Lagrange multipliers $\phi_{u}^{a}$, we obtain the representation,

$$
Z_{\text {mag }}=\int[\mathcal{D} \phi] e^{-S(\phi)}
$$

defined as in the Abelian case, but with $\phi$, being a pair of fields in the adjoint representation, and with a generating functional that now corresponds to the nonabelian case:

$$
\mathcal{W}(J)=-\ln \mathcal{Z}(J)
$$

\footnotetext{
${ }^{2}$ This could be verified, for example, by means of an appropriate transformation of the corresponding Lagrange multipliers.
} 
with

$$
\mathcal{Z}(J) \equiv \int[\mathcal{D} A] e^{-S_{g}(A)+\frac{i}{2} \int d^{4} x F_{\mu \nu}^{u}(y, x ; \mathcal{C}) J_{u}^{\mu \nu}(x)},
$$

where $S_{g}(A)$ is the gauge-fixed Yang-Mills action.

The vacuum energy $E_{0}$, defined as in the Abelian case, must be gauge invariant. This fact, naturally expected from the gauge invariance of the boundary conditions at $\Sigma$, may be verified explicitly as follows: under a gauge transformation associated with the group transformation $U(x)$, the shifted field strength transforms in the adjoint representation, with the induced group rotation evaluated at $y$. This change can be compensated by a parallel transport (also in the adjoint representation) of the Lagrange multipliers to the point $y$. Being that a unitary transformation, no non-trivial Jacobian is generated.

We also note that, had an Abelian theory been considered, we would have $F_{\mu \nu}(y, x ; \mathcal{C})=F_{\mu \nu}(x)$ and this calculation would have reduced to the one performed in the previous section.

Returning to the general nonabelian case, taking functional derivatives of $\mathcal{Z}(J)$ at $J=0$, we may generate field strength correlators,

$$
\left\langle F_{\mu_{1} \nu_{1}}^{u_{1}}\left(y, x_{1} ; \mathcal{C}_{1}\right) \ldots F_{\mu_{n} \nu_{n}}^{u_{n}}\left(y, x_{n} ; \mathcal{C}_{n}\right)\right\rangle
$$

which, due to colour conservation satisfy:

$$
\left\langle F_{\mu_{1} \nu_{1}}^{u}\left(y, x_{1} ; \mathcal{C}_{1}\right)\right\rangle=0
$$

and

$$
\frac{g^{2}}{4 \pi}\left\langle F_{\mu \nu}^{u_{1}}(y, x ; \mathcal{C}) F_{\rho \sigma}^{u_{2}}\left(y, x^{\prime} ; \mathcal{C}^{\prime}\right)\right\rangle=\frac{1}{4} \delta^{u_{1} u_{2}} F_{\mu \nu \rho \sigma}\left(x, x^{\prime}, y ; \mathcal{C}, \mathcal{C}^{\prime}\right) .
$$

It can also be shown that the correlator in (34) may only depend on $\mathcal{C}-\mathcal{C}^{\prime}$, the curve connecting $x$ to $x^{\prime}$.

To proceed, we recall that the SVM is based on the strong assumption that this correlator does not depend at all on $\mathcal{C}$ and $\mathcal{C}^{\prime}$. Moreover, the expectation value of the product of any number of shifted $F$ 's vanishes, while the product of an even number can be decomposed in terms of the sum of the different products that can be formed with just two field correlators. In other words, the assumptions of the SVM are tantamount to saying that the quantum fluctuations are controlled by an essentially Gaussian measure. To define the model, it is then sufficient to specify a quadratic $\mathcal{W}(J)$ functional, with the structure:

$$
\mathcal{Z}(J)=e^{-\frac{\pi}{8 g^{2}} \int d^{4} x d^{4} x^{\prime} J_{u}^{\mu \nu}(x) F_{\mu \nu ; \rho \sigma}\left(x, x^{\prime}\right) J_{u}^{\rho \sigma}\left(x^{\prime}\right)} .
$$


As a consequence,

$$
\mathcal{Z}_{\text {mag }}=\left[\int \mathcal{D} \phi e^{-\frac{\pi}{8 g^{2}} \int d^{4} x d^{4} x^{\prime} J_{\mu \nu}^{\Sigma}(x) F_{\mu \nu ; \rho \sigma}\left(x, x^{\prime}\right) J_{\rho \sigma}^{\Sigma}\left(x^{\prime}\right)}\right]^{N},
$$

where now $\phi$ refers to just a single pair of fields $\phi^{a}$ (without any colour index).

The correlator for two shifted field strengths is frequently parametrized as follows 3 :

$$
\begin{aligned}
F_{\mu \nu ; \rho \sigma}\left(x, x^{\prime}\right) & =\left(\delta_{\mu \rho} \delta_{\nu \sigma}-\delta_{\mu \sigma} \delta_{\nu \rho}\right) \kappa D\left(-z^{2}\right) \\
& +\frac{1}{2}\left[\partial_{\nu}\left(z_{\sigma} \delta_{\mu \rho}-z_{\rho} \delta_{\mu \sigma}\right)+\partial_{\mu}\left(z_{\rho} \delta_{\nu \sigma}-z_{\sigma} \delta_{\nu \rho}\right)\right](1-\kappa) D_{1}\left(-z^{2}\right)
\end{aligned}
$$

where $z \equiv x-x^{\prime}$.

Then, within the SVM, the Casimir energy is just $N$ times the one computed in some kind of generalized Abelian theory, namely, the one which comes from the modified kernel $F_{\mu \nu ; \rho \sigma}$. Note, in particular, that it contains a $D$-term, an object that is not present in the Abelian case (because of the absence of magnetic monopoles). This new term comes from the nonabelian character of the theory, and can be associated with the dual superconductor scenario [16] of the QCD vacuum, representing the effects of a chromomagnetic monopole condensate; it is crucial to derive the confining linear potential between heavy charges. The second term (Abelian part) contains the modified nonlocal kernel $D_{1}$, where perturbative effects dominate. We will discuss later specific fittings of $D$ and $D_{1}$ which are obtained from lattice data.

\section{Field representation of the Casimir energy}

An equivalent functional integral representation for the Casimir energy, previously expressed in terms of shifted field strength correlators, can be constructed in terms of Gaussian fields, albeit with non standard propagators. We have found that the most economical parametrization is in terms of a vector field $A_{\mu}$ and a pseudo-vector field $\Phi_{\mu}$ :

$$
F_{\mu \nu}=\partial_{\mu} A_{\nu}-\partial_{\nu} A_{\mu}+\epsilon_{\mu \nu \rho \sigma} \partial_{\rho} \Phi_{\sigma}
$$

where the new $\Phi$-dependent term represents the monopole sector of nonperturbative Yang-Mills. Similar field representations have been considered

\footnotetext{
${ }^{3}$ For a recent review see, for example, ref. [13].
} 
to discuss Wilson loops, also in the context of the method of field strength correlators [17].

Then the $\langle F F\rangle$ correlation function is now given by:

$$
\begin{aligned}
& \left\langle F_{\mu \nu}(x) F_{\rho \sigma}(y)\right\rangle= \\
& \quad=\left\langle\left(\partial_{\mu} A_{\nu}-\partial_{\nu} A_{\mu}\right)\left(\partial_{\rho} A_{\sigma}-\partial_{\sigma} A_{\rho}\right)\right\rangle+\epsilon_{\mu \nu \mu^{\prime} \nu^{\prime}} \epsilon_{\rho \sigma \rho^{\prime} \sigma^{\prime}} \partial_{\mu^{\prime}} \partial_{\rho^{\prime}}\left\langle\Phi_{\nu^{\prime}}(x) \Phi_{\sigma^{\prime}}(y)\right\rangle \\
& \quad \equiv \hat{O}_{\mu \nu, \rho \sigma}(G-S)^{\prime}+I_{\mu \nu, \rho \sigma} \partial^{2} S,
\end{aligned}
$$

where $\left\langle A_{\mu}(x) \Phi_{\nu}(y)\right\rangle=0$,

$$
\left\langle A_{\mu}(x) A_{\nu}(y)\right\rangle=\delta_{\mu \nu} G\left(z^{2}\right) \quad, \quad\left\langle\Phi_{\mu}(x) \Phi_{\nu}(y)\right\rangle=\delta_{\mu \nu} S\left(z^{2}\right) .
$$

The prime represents derivative with respect to $z^{2}$, and we used the notation:

$$
\begin{gathered}
I_{\mu \nu, \rho \sigma} \equiv\left(\delta_{\mu \rho} \delta_{\nu \sigma}-\delta_{\mu \sigma} \delta_{\nu \rho}\right) \\
\hat{O}_{\mu \nu, \rho \sigma}=2\left\{\partial_{\nu}\left[\delta_{\mu \rho} z_{\sigma}-\delta_{\mu \sigma} z_{\rho}\right]+\partial_{\mu}\left[\delta_{\nu \sigma} z_{\rho}-\delta_{\nu \rho} z_{\sigma}\right]\right\} .
\end{gathered}
$$

Then, to reproduce the lattice parametrization of (37), one can make the identifications,

$$
\partial^{2} S=\kappa D \quad, \quad G^{\prime}-S^{\prime}=(1-\kappa) D_{1} / 4,
$$

which can be solved by imposing the following equations,

$$
\tilde{S}=-\kappa \frac{\tilde{D}}{k^{2}} \quad, \quad \tilde{G}=-\kappa \frac{\tilde{D}}{k^{2}}+(1-\kappa) \tilde{H} \quad, \quad H^{\prime}=D_{1} / 4
$$

In other words, the (only) nontrivial propagators are,

$$
\begin{aligned}
\left\langle A_{\mu}(x) A_{\nu}(y)\right\rangle & =-\int \frac{d^{4} k}{(2 \pi)^{4}}\left[\kappa \tilde{D}+(1-\kappa) k^{2} \tilde{H}\right] \frac{\delta_{\mu \nu}}{k^{2}} e^{i k \cdot(x-y)} \\
\left\langle\Phi_{\mu}(x) \Phi_{\nu}(y)\right\rangle & =-\int \frac{d^{4} k}{(2 \pi)^{4}}[\kappa \tilde{D}] \frac{\delta_{\mu \nu}}{k^{2}} e^{i k \cdot(x-y)} .
\end{aligned}
$$

We see that $\tilde{D}$ and $k^{2} \tilde{H}$ became associated with 'structure functions' for the propagators of the $A_{\mu}$ and $\Phi_{\mu}$ fields. where

Then, the partition function (함) can be also represented by, $\mathcal{Z}_{m a g}=\mathcal{Z}_{A, \Phi}^{N}$,

$$
\mathcal{Z}_{A, \Phi}=\int \mathcal{D} \phi \mathcal{D} A \mathcal{D} \Phi e^{-S_{e}[A]-S_{m}[\Phi]+\frac{i}{2} \int d^{4} x J_{\mu \nu}^{\Sigma}\left(\partial_{\mu} A_{\nu}-\partial_{\nu} A_{\mu}+\epsilon_{\mu \nu \rho \sigma} \partial_{\rho} \Phi_{\sigma}\right)} .
$$


Path integrating over the Lagrange multipliers $\phi$, we get the constraints,

$$
F_{i j} n^{j}=\left(\partial_{i} A_{j}-\partial_{j} A_{i}+\epsilon_{i j \rho \sigma} \partial_{\rho} \Phi_{\sigma}\right) n^{j}=0,
$$

namely, the Yang-Mills fields Casimir energy, due to Gaussian dominance in the shifted field strength correlators, can be written as $N$ times the one obtained for a field theory with action $S_{e}[A]+S_{m}[\Phi]$. Here, both $S_{e}[A]$ and $S_{m}[\Phi]$ are (typically) nonlocal versions of Maxwell-like actions; for instance, $S_{m}[\Phi] \sim \int d^{4} x G_{\mu \nu}\left[\tilde{D}^{-1}\left(\partial^{2}\right)\right] G_{\mu \nu}$, with $G_{\mu \nu}=\partial_{\mu} \Phi_{\nu}-\partial_{\nu} \Phi_{\mu}$. We shall assume that a Lorentz gauge-fixing condition for $A_{\mu}$ and $\Phi_{\mu}$ is implicitly included in the functional integral measure.

Then, we have,

$$
\mathcal{Z}_{A, \Phi}=\int[\mathcal{D} A \mathcal{D} \Phi]_{m a g} e^{-S_{e}[A]-S_{m}[\Phi]}
$$

where the path integral is carried over fields $A_{\mu}, \Phi_{\mu}$ satisfying the condition (47). Note that the original constraints yield boundary conditions corresponding to a perfect conductor of magnetic charges, while the field strength receives contributions from both 'electric monopole' $(A)$ and 'magnetic monopole' $(\Phi)$ sectors.

\section{Analysis of the gluon contribution to the Casimir bag energy}

For the case of pure $S U(3)$ 4, typical ansatze for $D$ and $D_{1}$ are (see ref. [14]):

$$
D\left(-z^{2}\right)=\frac{G_{2}}{24} \frac{27}{64} a^{-2} \int d^{4} k \frac{k^{2}}{\left(k^{2}+(3 \pi / 8 a)^{2}\right)^{4}} \exp (i k z),
$$

and

$$
D_{1}\left(-z^{2}\right)=\left(z^{2}\right)^{-2} \int_{0}^{z^{2}} d v 2 v D(-v) .
$$

We note that for large $z^{2}, D \sim \exp \left(-\frac{3 \pi|z|}{8 a}\right)$ and,

$$
D_{1}\left(-z^{2}\right) \sim\left(z^{2}\right)^{-2}
$$

\footnotetext{
${ }^{4}$ The effect of dynamical fermions on field strength correlators can be studied on the lattice, see ref. [20]. The model parameters get renormalized, however, our general analysis is left unchanged.
} 
Although a pure $S U(3)$ lattice calculation fixes the parameters to $\kappa=0.74$, $a=0.35 \mathrm{fm}$ and $G_{2}=(496 \mathrm{MeV})^{4}$, see [11, 14] and references therein, it is interesting to study what happens when $\kappa$ goes from 0 to 1 .

When $\kappa=0$, there is just one propagating field,

$$
\left\langle A_{\mu}(x) A_{\nu}(y)\right\rangle=-\tilde{H} \delta_{\mu \nu},
$$

the leading contribution to $D_{1}$ is perturbative, $D_{1} \sim 1 / z^{4}$, and from (44) we see that $H \sim 1 / z^{2}$. Thus, the leading behaviour of the $A_{\mu}$ propagator is $\sim\left(1 / k^{2}\right) \delta_{\mu \nu}$. As this corresponds to the usual behaviour of $Q E D(4)$, the Casimir effect at $\kappa=0$ is expected to be repulsive for a bag, and after multiplying by $N=8, E_{c} \approx+0.7 / R$.

Then, for $\kappa$ close to zero, the usual result for the simplified model where the Casimir bag energy is computed considering the gluon modes as photonlike fields is recovered. Of course, the lattice fitting value $\kappa=0.74$ is far from that regime, and it comes as no surprise that the Casimir bag energy for the simplified model, after adding contributions coming from center of mass fluctuations, is far from the required value to adjust hadrons.

Since $\kappa$ is in fact closer to one than to zero, let us analyze the opposite regime, when $\kappa=1$. The field propagators are now given by,

$$
\left\langle A_{\mu}(x) A_{\nu}(y)\right\rangle=\left\langle\Phi_{\mu}(x) \Phi_{\nu}(y)\right\rangle=-\tilde{D} \frac{\delta_{\mu \nu}}{k^{2}} .
$$

We can also write,

$$
S_{e}[A]+S_{m}[\Phi]=\frac{1}{2}\left(\begin{array}{ll}
A_{\mu} & \Phi_{\mu}
\end{array}\right) \delta_{\mu \nu}\left(\partial^{2} / \tilde{D}\right)\left(\begin{array}{cc}
1 & 0 \\
0 & 1
\end{array}\right)\left(\begin{array}{c}
A_{\nu} \\
\Phi_{\nu}
\end{array}\right)
$$

(where in $\tilde{D}$ we have replaced $k^{2}$ by $-\partial^{2}$ ).

Now, we note that (49) implies,

$$
\tilde{D} \propto \frac{k^{2}}{\left(k^{2}+\Lambda^{2}\right)^{4}} \quad, \quad \Lambda=(3 \pi / 8 a),
$$

so that the kinetic operator in (54) is proportional to,

$$
\delta_{\mu \nu} I_{2 \times 2}\left(-\partial^{2}+\Lambda^{2}\right)^{4}
$$

and the Casimir energy implied by (48) is four times the one for a kinetic operator,

$$
\delta_{\mu \nu} I_{2 \times 2}\left(-\partial^{2}+\Lambda^{2}\right) .
$$

We see that the $A$ and $\Phi$ propagators are both infrared suppressed, since the associated modes are in fact massive. The lattice adjusted value of $a=$ 
$0.35 \mathrm{fm}$, corresponds to $\Lambda \sim 660 \mathrm{Mev}^{-1}$. Casimir energies associated with massive modes at this scale, have been precisely discussed in refs. [18, 19], and are strongly suppressed, for a typical hadron radius, when compared with contributions of order $\sim 1 / R$. Typical values of $R$ for the smaller hadrons are $\sim 2 / 3 \mathrm{fm}, \sim 1 \mathrm{fm}\left(1 \mathrm{fm} \approx \frac{1}{200} M e v^{-1}\right)$.

It is interesting to note that the field representation we have obtained here is quite similar to the simple infrared modified bag model introduced in ref. [18]. In that reference, information associated with a mass scale $\Lambda$ was encoded in a quadratic Abelian field theory, containing an effective gluon propagator, thus discussing possible effects of nonperturbative infrared physics on Casimir energy calculations.

With regard to the equivalent field theory discussed in the present work, three important remarks are in order: i) the field theory has been derived rather than assumed: its quadratic nature is a direct consequence of the Gaussian dominance in shifted field strength correlators, the 'hard' part of the calculation, namely, the generally nonlocal kernels in the kinetic terms, is borrowed from lattice data; ii) as it is based on field strength correlators, the formulation is $\mathrm{SU}(3)$ gauge invariant, as well as the defined Casimir energy and the nonperturbative information encoded in the kernels; iii) It contains a vector and a pseudovector field, representing electric and magnetic monopole sectors; the field strength receives contributions from both.

For the lattice value of $\kappa=0.74$, the Casimir effect will be presumably closer to what happens at $\kappa=1$, where the effect is suppressed with respect to contributions of order $\sim 1 / R$, than to the value of $\sim+0.7 / R$, at $\kappa=0$, implied by "photon-like" gluons. So that the $R^{-1}$ term in the bag energy is expected to be attractive, dominated by center of mass fluctuations $-Z_{c m} / R$, $Z_{c m} \sim 1$.

\section{Conclusions}

In order to fit properly hadron spectroscopy, the MIT bag model requires a term in the bag energy of the form $-Z / R$, with $Z$ of order one. As it is well known, when Casimir energy calculations are done by means of the crudest simplification, considering 'photon-like' gluons, the effect turns out to be $\sim+0.7 / R$, with the wrong sign. Improvement on this disappointing result has been, for many years, blocked by the fact that a full nonabelian calculation would be extremely difficult to perform.

In this article, we have introduced a middle way to approach the problem, namely, to take the most relevant nonabelian effects into account.

Using a technique based on auxiliary fields living on the bag boundary, 
to impose the bag constraints, we have been able to express the Casimir bag energy in terms of shifted field strength correlators in $S U(3)$. Of course, the obtained Casimir energy representation is invariant under $S U(3)$ gauge transformations.

As lattice simulations show, the shifted field strength correlators possess Gaussian dominance and can be parametrized in terms of two structure functions fitted with the lattice data. This is the so called stochastic vacuum model of QCD and it consistently describes short distance perturbative aspects, such as asymptotic freedom, as well as large distance nonperturbative ones, such as confinement. Therefore, it is a natural approximation scheme to discuss whether the simple model that treats gluon modes as free Abelian massless fields can be applied, and if not, what are the consequences of taking nonperturbative effects into account.

Gaussian dominance enables the introduction of an equivalent quadratic field representation in terms of vectorial and pseudovectorial effective fields, representing electric and magnetic monopole degrees of freedom, respectively. This representation contains two generally nonlocal kernels, whose typical ansatze contains a $\kappa$ parameter weighting the nonabelian nature of gluon fields. For $\kappa$ close to zero, the effective model is dominated by perturbative physics, so that in this case a "photon-like" gluon model would be obtained, together with the associated Casimir energy, $\sim+0.7 / R$.

However, the lattice adjusted value is $\kappa=0.74$, closer to $\kappa=1$, a value where the equivalent Gaussian fields have a mass gap $\Lambda=660 \mathrm{Mev}$ and the Casimir energy for typical hadron radius is strongly suppressed. Therefore, the Casimir energy of pure $S U(3)$ theory is expected to be suppressed, when compared with attractive center of mass contributions, $-Z_{c m} / R, Z_{c m}$ of order one. An important point is that the infrared suppression of propagators, in our equivalent field representation, is $\mathrm{SU}(3)$ gauge invariant: the fields represent effective modes with a nonperturbative gap, associated with $\mathrm{SU}(3)$ gauge invariant structure functions of shifted field strength correlators.

The predicted bag energy term is, therefore, $-Z / R, Z \sim Z_{c m} \sim 1$. To the best of our knowledge, this is the first controlled estimation of the $1 / R$ term in the bag energy, and it turns out to comply with hadron phenomenology.

\section{Acknowledgements}

The Conselho Nacional de Desenvolvimento Científico e Tecnológico (CNPq) and the Fundação de Amparo à Pesquisa do Estado do Rio de Janeiro (FAPERJ) are acknowledged for the financial support. C. D. F. acknowledges support by CONICET and ANPCyT (Argentina). 


\section{References}

[1] T. DeGrand, R. L. Jaffe, K. Johnson and J. Kiskis, Phys. Rev. D12, 2060 (1975).

[2] T. H. Boyer, Phys. Rev. 174, 1764 (1968).

[3] B. Davies, J. Math. Phys. 13, 1324 (1972).

[4] C. M. Bender and P. Hays, Phys. Rev. D14, 2622 (1976).

[5] K. A. Milton, Phys. Rev. D22, 1441 (1980).

[6] K. A. Milton, Phys. Rev. D27, 439 (1983).

[7] K. A. Milton, The Casimir Effect, World Scientific, Singapore (2001).

[8] J. F. Donoghue, E. Golowich and B. R. Holstein, Dynamics of the Standard Model, Cambridge University Press, Cambridge (1992).

[9] K. A. Milton, Phys. Rev. D22, 1444 (1980); K. A. Milton, Phys. Rev. D25, 3441(E) (1982).

[10] J. F. Donoghue and K. Jhonson, Phys. Rev. D21, 1975 (1980);

C. W. Wong, Phys. Rev. D24, 1416 (1981);

K. F. Lui and C. W. Wong, Phys. Lett. B113, 1 (1982).

[11] A. Di Giacomo and H. Panagopoulos, Phys. Lett. B285, 133 (1992);

H. G. Dosch, E. Ferreira and A. Krämer, Phys. Rev. D50, 1992 (1994).

[12] H. G. Dosch, Phys. Lett. B190, 177 (1987);

H. G. Dosch and Yu A. Simonov, Phys. Lett. B205, 339 (1988);

Yu A. Simonov, Nucl. Phys. B307, 512 (1988).

[13] A. Di Giacomo, H. G. Dosch, V. I. Shevchenko, Yu. A. Simonov; Phys. Rept. 372, 319 (2002).

[14] O. Nachtmann, Lectures given at Banz (Germany) 1993 and at Schladming (Austria) 1996, Report-no: HD-THEP-96-38, hep-ph/9609365.

[15] R. Golestanian and M. Kardar, Phys. Rev. A58, 1713 (1998).

[16] G. t'Hooft, Nucl. Phys. B138, 1 (1978); Nucl. Phys. B190, 455 (1981).

[17] D. Antonov, String Nature of Confinement in (Non-)Abelian Gauge Theories, Ph.D. thesis at the Humboldt University of Berlin (1999), Surveys High Energ.Phys. 14, 265 (2000), hep-th/9909209. 
[18] L. E. Oxman, N. F. Svaiter and R. L. P. G. Amaral, Phys. Rev. D72, 125007 (2005).

[19] M. Bordag, E. Elizalde, K. Kirsten and S. Leseduarte, Phys. Rev. D56, 4896 (1997).

[20] M. D’Elia, A. Di Giacomo, E. Meggiolaro; Phys. Lett. B408, 315 (1997). 